\title{
Personalization of Cubic Hermite Meshes for Efficient Biomechanical Simulations
}

\author{
Pablo Lamata ${ }^{1}$, Steven Niederer ${ }^{1}$, David Barber ${ }^{2}$, David Norsletten ${ }^{1}$, \\ Jack Lee ${ }^{1}$, Rod Hose ${ }^{2}$, and Nic Smith ${ }^{1}$ \\ 1 Computing Laboratory, University of Oxford, UK \\ \{pablo.lamata, steven.niederer, david.norsletten, \\ jack.lee,nic.smith\}@comlab.ox.ac.uk \\ 2 Department of Cardiovascular Science, University of Sheffield, UK \\ $\{$ d.barber, d.r.hose\}@sheffield.ac.uk
}

\begin{abstract}
Cubic Hermite meshes provide an efficient representation of anatomy, and are useful for simulating soft tissue mechanics. However, their personalization can be a complex, time consuming and labourintensive process. This paper presents a method based on image registration and using an existing template for deriving a patient-specific cubic Hermite mesh. Its key contribution is a solution to customise a Hermite continuous description of a shape with the use of a discrete warping field. Fitting accuracy is first tested and quantified against an analytical ground truth solution. To then demonstrate its clinical utility, a generic cubic Hermite heart ventricular model is personalized to the anatomy of a patient, and its mechanical stability is successfully tested. The method achieves an easy, fast and accurate personalization of cubic Hermite meshes, constituting a crucial step for the clinical adoption of physiological simulations.
\end{abstract}

\section{Introduction}

Computational physiology provides tools to quantitatively describe physiological behaviour across a range of time scales and anatomical levels using mathematical and computational models [1,2]. The heart is arguably the most advanced current exemplar of this approach 34, and ongoing developments now have the potential to provide a significant impact in the management of cardiovascular diseases. One of the key challenges in fulfilling this potential is the personalization of models to represent the clinical status of a patient. This work focuses on the efficient and automated development of patient-specific geometrical description of organs for biomechanical simulations.

A computational model requires the geometrical description of the solution domain where material constitutive equations are solved. The most popular choice is linearly interpolated tetrahedral meshes, mainly due to its conceptual simplicity and availability of tools for an automatic mesh generation [5]. Nevertheless, they introduce significant numerical error in the solution of the displacements in the soft tissue deformation problem 6]. Alternatively, cubic Hermite meshes are

T. Jiang et al. (Eds.): MICCAI 2010, Part II, LNCS 6362, pp. 380-387, 2010.

(C) Springer-Verlag Berlin Heidelberg 2010 
an efficient representation of the geometrical state of an organ, and a more suitable choice for biomechanical simulations compared to tetrahedral meshes [6]. Another important requirement is mesh robustness, related to the convergence of simulation results in changing physiological conditions. For these reasons, cubic Hermite meshes are a popular choice for the simulation of heart mechanical deformations 789. Nevertheless, construction of these meshes can be a complex, time consuming and labour-intense process. There is thus a need for a fast, accurate, robust and easy to use cubic Hermite personalization method.

There are two broad approaches for the personalization of geometrical meshes: construction from segmented images [510] or customization from an existing mesh model [1110]. Whereas the literature for linear meshes is extensive [5], its translation to Hermite meshes is not straightforward. The change of interpolation scheme, from linear to Hermite functions, requires a completely different meshing strategy, like the adaptation of the Iterative Closest Point proposed in [10]. In a Hermite mesh shape is interpolated, not only from the 3D Cartesian coordinates of nodes, but also from the derivatives of shape versus local finite element (FE) coordinates. This enables a compact representation, but results in a complex mesh construction and customization. This article presents an image registration based solution for cubic Hermite mesh personalization.

\section{Material and Methods}

The proposed personalization method combines a fast binary image registration with a cubic Hermite warping technique. A schematic illustration of the complete process is provided in Fig [1.

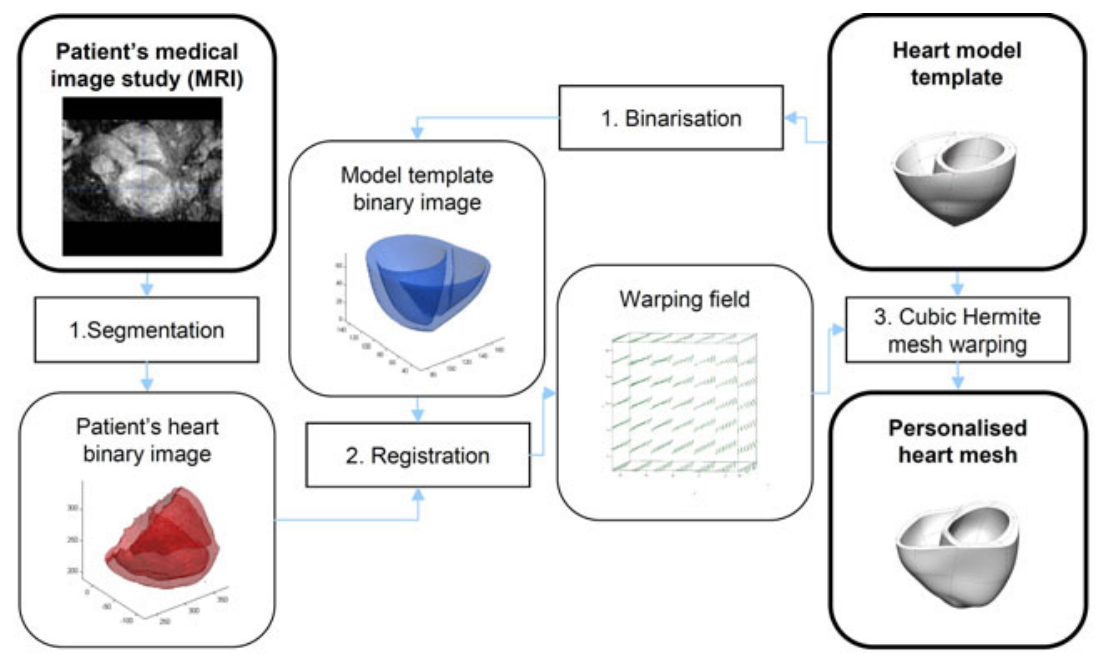

Fig. 1. Dataflow designed to generate patient specific cubic Hermite meshes 


\subsection{Image Registration}

A binary image registration technique using a fast optical flow algorithm proposed in 11] is chosen for its robustness, accuracy and computational efficiency. It requires a preliminary segmentation of the patient's anatomy, which in the case of heart ventricles from static MRI or CT is a quite mature field of research. Discrete values of the warping field between the two binary images are defined at nodes of a regular hexahedral grid superimposed on the binary image of the template shape. The two main parameters of the registration technique are the spacing between nodes $D$ and the smoothing weighting factor $\lambda$ (based on a Tikhonov regularisation of the linear least squares problem). Registration is initialised by aligning the principal axis of the shapes after a Principal Component Analysis of the 3D coordinates of each shape.

\subsection{Cubic Hermite Mesh Warping}

A cubic Hermite mesh is a set of 3D FE that uses Hermite interpolation functions. Mesh nodes have both coordinate values and derivatives (single, double and triple cross derivatives) in order to encode a $C^{1}$ shape. Let $u\left(\xi_{1}, \xi_{2}, \xi_{3}\right)$ be a shape defined in the Cartesian space as a function of the material coordinates $\xi$. The four 1D Hermite interpolation basis functions are described in (1), and interpolation in a line element $u(\xi)$ is given by a linear combination of these four basis functions (2). This scheme can be extended to 3D as illustrated in Fig,2, $u\left(\xi_{1}, \xi_{2}, \xi_{3}\right)$ is then interpolated from a total of 192 variables in each element.

$$
\begin{gathered}
\psi_{1}^{0}(\xi)=1-3 \xi^{2}+2 \xi^{3} ; \psi_{2}^{0}(\xi)=\xi^{2}(3-2 \xi) \\
\psi_{1}^{1}(\xi)=\xi(\xi-1)^{2} ; \psi_{2}^{1}(\xi)=\xi^{2}(\xi-1) \\
u(\xi)=\psi_{1}^{0}(\xi) u_{1}+\psi_{2}^{0}(\xi) u_{2}+\psi_{1}^{1}(\xi) d u /\left.d \xi\right|_{1}+\psi_{2}^{1}(\xi) d u /\left.d \xi\right|_{2}
\end{gathered}
$$

Warping a cubic Hermite mesh therefore implies calculation of a deformed state of its three coordinate fields $(x, y, z)$. The total number of degrees of freedom is therefore $N_{d o f}=3 \times 8 \times n=24 n$, being $n$ the number of nodes of the mesh. Note that this number will be slightly smaller if the mesh has collapsed elements, and slightly bigger if there are discontinuities modelled with different versions of node values. For further details about these meshes see [10].

The solution for the warping of a cubic Hermite mesh is the core contribution of this work. This third step in Fig 1 calculates the optimal description of a warped shape with Hermite interpolation functions. This is built on three basic concepts. First, it is important to realise that the warping of a FE requires knowing the warping field throughout the complete domain, and not only in the local neighbourhood of mesh nodes. Second, the solution uses a FEM technique for finding an optimal representation of a field in a domain, i.e. it uses a variational formulation of the problem based on a dot product of functions. And third, a numerical technique is required to handle continuous domains with computer discrete representations, i.e. a numerical integration method is used to calculate the dot product of functions. 


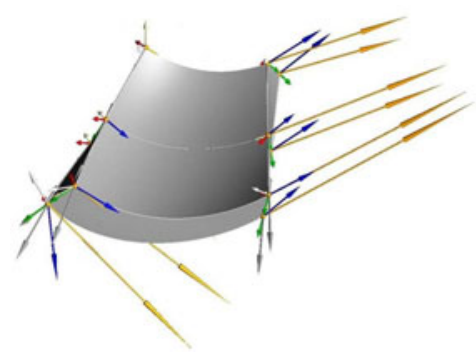

(a)

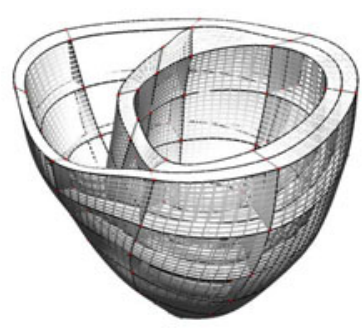

(b)

Fig. 2. Cubic Hermite meshes. (a) A two element mesh, showing the vectors corresponding to the 7 derivatives at each node; (b) template of truncated left and right ventricles of the heart. This complex shape is represented with only 112 cubic elements (cubes with black lines) and 183 nodes.

The central idea is that warping of a cubic Hermite mesh is an addition of a FE description of a warping field. It requires finding the adequate FE description, with a set of $N_{d o f}$ variables as described before, of the warping field. And this description is then added to the corresponding $N_{\text {dof }}$ nodal variables describing the 3 coordinate fields, i.e. the shape. Let us define a variational problem for finding the $C^{1}$ continuous function $g$ that approximates $w$, one of the three components of the warping field $W$ in the domain defined by the cubic Hermite template $\Theta_{T}$. Let $g$ be formulated as a linear combination of the set of $N_{d o f} / 3$ basis functions $\phi_{T}$ of the mesh. Note that each $\phi_{T}$ is a combination of 3D Hermite basis from adjacent elements in order to enforce the continuity of the function and its derivatives. Let us introduce the definition of a dot product of functions:

$$
\left(g, \phi_{j}\right)=\int_{\Theta} g \cdot \phi_{j}=\int_{\Theta} \sum_{i} c_{i} \phi_{i} \cdot \phi_{j}=\sum_{i} c_{i} \int_{\Theta} \phi_{i} \cdot \phi_{j}
$$

Finding $g$ becomes the problem of finding the $c_{i}$ coefficients (or nodal values) which satisfy a set of $N_{d o f} / 3$ equations, one for each basis $\phi_{T}$ :

$$
\begin{aligned}
& \left(g, \phi_{j}\right)=\left(w, \phi_{j}\right) \forall \phi_{j} \\
& \sum_{i} c_{i} \int_{\Theta_{T}} \phi_{i} \cdot \phi_{j}=\int_{\Theta_{T}} w \cdot \phi_{j} \\
& M x=b
\end{aligned}
$$

where vector $x$ is the set of $N_{d o f} / 3$ coefficients $c_{i}$, and integrals required to calculate matrix $M$ and vector $b$ are computed using Gaussian Quadrature. Calculi of vector $b$ uses order 4 , and cubic interpolation of the warping field is used to calculate the data (deformation field) at Gauss Points. The linear system of the fitting process requires the solution of a sparse matrix system. This matrix is symmetric and positive definite, allowing the use of fast, low-memory solvers such as Conjugate Gradients. 


\section{Results}

\subsection{Analytical Workbench for Accuracy and Robustness Analysis}

Accuracy and sensitivity to parameters are analysed using a virtual workbench with known analytical solutions. Three experiments study the accuracy of (1) proposed warping scheme, step 3 in Fig 1, (2) the binary image registration, step 2 in Fig.1, and (3) the concatenation of the two processes. Template cylinders are built with dimensions 30,10 and $5 \mathrm{~mm}$ in length, outer and internal radius, and with 24, 81 and 192 elements. Warped versions of these cylinders are generated under two known warping fields $W_{1}$ and $W_{2}$, see Fig 3. Shape error is calculated in each element as the integral of the RMS error between warped coordinates field and their ground truth. An order 5 Gaussian Quadrature volume-weighted integration of error is used, which is independent of the mesh discretisation resolution and topology.

The warping step is analysed by using a perfect solution of the registration step, obtained by sampling the ground truth warping fields. This experiment is repeated for $W_{1}$ and $W_{2}$, for the three cylinder mesh resolutions and for a range of node spacings in the discretisation of the warping field $\left(S_{W}\right.$, with 9 values from 1 to $5 \mathrm{~mm}$ in steps of $0.5 \mathrm{~mm}$ ). Average shape error is $5.5 e^{-3}$ and $5.5 e^{-2} \mathrm{~mm}^{2}$ for $W_{1}$ and $W_{2}$ respectively, and the dependence with the two factors (mesh resolution and $S_{W}$ ) is illustrated in Fig 4

The registration step is analysed by comparison of obtained warping fields with their analytical expression. Binary images are generated from the Ground Truth shapes. Registration is performed for each $W_{1}$ and $W_{2}$, for a total of 49 binary image resolutions (from $0.2 \mathrm{~mm}$ to $5 \mathrm{~mm}$ in steps of $0.1 \mathrm{~mm}$ ), and for 9 values of node spacing ( $D$ from 2 to 10 voxels). The smoothing coefficient $\lambda$ is set automatically by an empirical theorem proposed in [11. Average registration volume-weighted error is 1.86 and $0.69 \mathrm{~mm}$ RMS for $W_{1}$ and $W_{2}$ respectively, results are shown in Fig 5 .

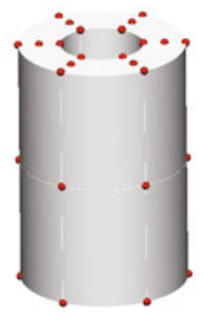

(a) Template

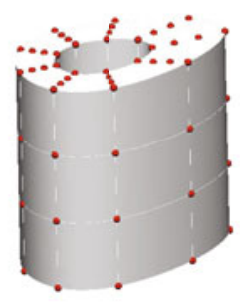

(b) $W_{1}=\left(x^{3}, x^{3}, 1\right)$

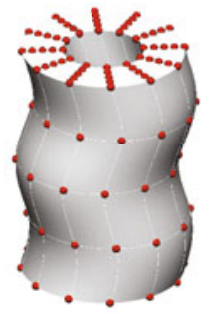

(c) $W_{2}=(\sin (z), 0,0)$

Fig. 3. GroundTruth. All three shapes (template, customised by $W_{1}$ and customised by $\left.W_{2}\right)$ are created with three resolutions (24, 81 and 192 elements). 


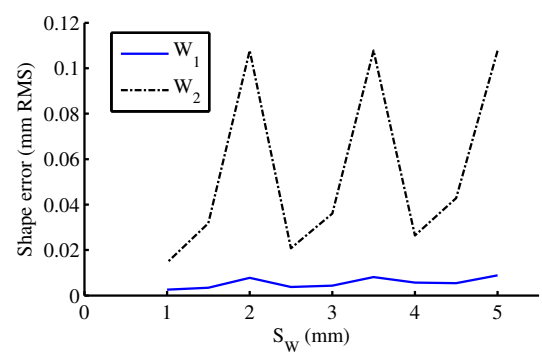

(a)

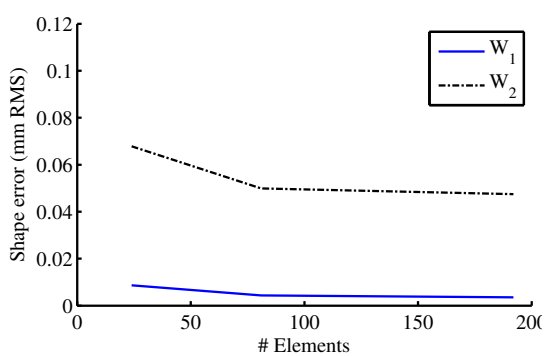

(b)

Fig. 4. Error introduced in the warping of cubic Hermite meshes using a discrete version of the ideal warping fields $W_{1}$ and $W_{2}$. (a) Dependence on sampling resolution, $\left(S_{W}\right)$, averaging results with three mesh resolutions. (b) Dependence on mesh resolution, averaging results with nine sampling resolutions.

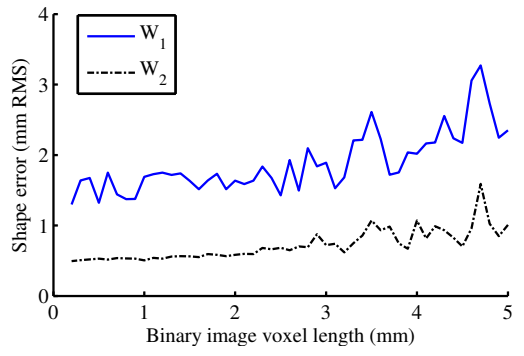

(a)

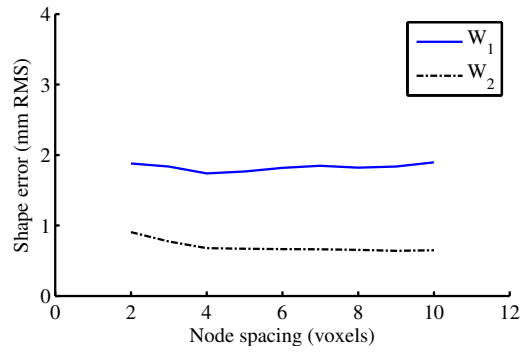

(b)

Fig. 5. Registration error (volume-weighted integral) for warping fields $W_{1}$ and $W_{2}$. (a) Dependence on image resolution, averaging results with 9 node spacings. (b) Dependence on node spacing, averaging results with 49 image resolutions.

The previous two steps are concatenated, and template cylinder meshes are warped with the result of binary image registrations. The mesh resolution of 81 elements is chosen for this experiment, since higher resolutions did not introduce any significant improvement in accuracy, see Fig $4 \mathrm{~b}$. The analysis is repeated for $W_{1}$ and $W_{2}$, for all 49 binary image resolutions (from $0.2 \mathrm{~mm}$ to $5 \mathrm{~mm}$ in steps of $0.1 \mathrm{~mm}$ ), and for 9 node spacing (from 2 to 10 voxels). The average shape error for $W_{1}$ and $W_{2}$ is 1.90 and $0.702 \mathrm{~mm}$ RMS respectively, an increment of roughly a $1.5 \%$ with respect to the registration error. The dependence on binary resolution and node spacing is the same as reported for the registration step, see Fig 5 .

\subsection{Clinical Case}

A patient specific cubic Hermite mesh of heart ventricles is constructed following the process of Fig 1. The template mesh chosen is the result of fitting a mesh to the anatomy of a first patient following the methodology described in [10] and 
illustrated in Fig 2, A T1 MRI heart study of a second patient (0.88x0.88x0.75 $\mathrm{mm}$ voxel resolution) is manually segmented and truncated just underneath of the opening of valve planes after vertical alignment. The agreement between segmentation and resulting cubic Hermite mesh is shown in Fig 6, and the average distance between the two surfaces is measured as $1.32 \mathrm{~mm}$. The process from image segmentation to mesh fitting for this biventricular dataset of $90^{3}$ voxels requires about one minute. Mechanical stability of personalised geometry is successfully tested by simulating a passive inflation and an isochronous active contraction of the heart following the methods described in [7].
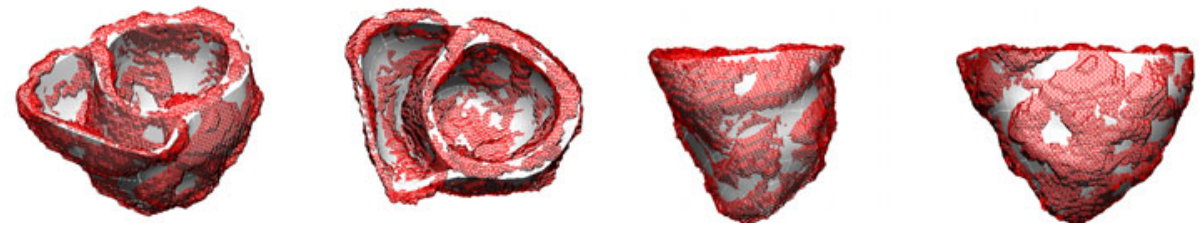

Fig. 6. Shape personalization result. Comparison of the isosurface of the binary manual segmentation (red wireframe) to the Cubic Hermite mesh (white solid).

\section{Discussion}

Cubic Hermite mesh warping requires the addition of an adequate representation of the warping field in nodal values and derivatives. The proposed solution finds an optimal description of this field, leading to reasonable accurate results.

Results show the importance of the registration step, which is limited by image resolution. Interpolation errors also become significant when the topology of the mesh is not able to represent the warped shape. These cases require an interpolation order higher than cubic or higher element refinement, such as case $W_{2}$. An inherent limitation of proposed approach is that binary registration only aligns the surfaces of models, interpolating the warping inside. This is a valid approach for vessels and computational fluid dynamics [11, and preliminary results suggest that this is also adequate for the flat walls of the heart.

The proposed method is its simpler and more robust compared to the state of the art alternatives, the "host mesh" technique 10 or the mesh generation by fitting Hermite surfaces from a linear scaffold [10]. Proposed method is fast and requires minimal user interaction. In comparison, the two alternative methods greatly depend on users expertise, and it can take hours of manual interaction and fitting to get satisfactory results. A second advantage is that, because it uses a voxelized description of the shape and not a selection of pairs of control points and degrees of freedom as required in a "host mesh" technique, it is immune to subjective and sometimes arbitrary selection of parameters. Finally, warping an existing high quality template mesh under a smoothness constraint is a reasonable warranty of simulation stability in the resulting mesh. This does not occur using a mesh generation from an arbitrary initial linear mesh [10]. Further experiments with higher number of cases are nevertheless required to 
generalise and accept these desirable properties. Future works will also address the characterization and development of a metric of mechanical stability of cubic Hermite meshes, a metric to be optimised during the personalization process.

\section{Conclusion}

Proposed method achieves an easy, fast and accurate personalization of cubic Hermite meshes, constituting a crucial step for the clinical adoption of biomechanical physiological simulations.

\section{References}

1. Hunter, P.J., Crampin, E.J., Nielsen, P.M.F.: Bioinformatics, multiscale modeling and the IUPS Physiome Project. Brief Bioinform 9(4), 333-343 (2008)

2. Lee, J., Niederer, S., Nordsletten, D., Le Grice, I., Smail, B., Kay, D., Smith, N.: Coupling contraction, excitation, ventricular and coronary blood flow across scale and physics in the heart. Phil. Trans. R Soc. A 367(1896), 2311-2331 (2009)

3. Smith, N.P., Nickerson, D.P., Crampin, E.J., Hunter, P.J.: Multiscale computational modelling of the heart. Acta Numerica 13(1), 371-431 (2004)

4. Bassingthwaighte, J., Hunter, P., Noble, D.: The Cardiac Physiome: perspectives for the future. Experimental Physiology 94(5), 597-605 (2009)

5. Löhner, R.: Automatic unstructured grid generators. Finite Elem. Anal. Des. 25(12), 111-134 (1997)

6. Pathmanathan, P., Whiteley, J.P., Gavaghan, D.J.: A comparison of numerical methods used for finite element modelling of soft tissue deformation. The Journal of Strain Analysis for Engineering Design 44(5), 391-406 (2009)

7. Niederer, S.A., Smith, N.P.: The role of the Frank Starling law in the transduction of cellular work to whole organ pump function: A computational modeling analysis. PLoS Comput. Biol. 5(4), e1000371 (2009)

8. Kerckhoffs, R.C., McCulloch, A.D., Omens, J.H., Mulligan, L.J.: Effects of biventricular pacing and scar size in a computational model of the failing heart with left bundle branch block. Medical Image Analysis 13(2), 362-369 (2009)

9. Wang, V.Y., Lam, H., Ennis, D.B., Cowan, B.R., Young, A.A., Nash, M.P.: Modelling passive diastolic mechanics with quantitative mri of cardiac structure and function. Medical Image Analysis 13(5), 773-784 (2009)

10. Fernandez, J., Mithraratne, P., Thrupp, S., Tawhai, M., Hunter, P.: Anatomically based geometric modelling of the musculo-skeletal system and other organs. Biomechanics and Modeling in Mechanobiology 2, 139-155 (2004)

11. Barber, D., Oubel, E., Frangi, A., Hose, D.: Efficient computational fluid dynamics mesh generation by image registration. MedIA 11(6), 648-662 (2007) 